\title{
Acute Oculogyric Crisis in a Patient Taking Clebopride
}

\author{
Woojun Kim, Kwang-Soo Lee, Yeong-In Kim and Joong-Seok Kim
}

\begin{abstract}
We present a 21-year-old woman with an oculogyric crisis following the administration of clebopride. Based on the temporal relationship of the administration of clebopride to the occurrence of the event and the absence of other possible etiologies, clebopride is implicated as the cause of this acute oculogyric crisis, despite the fact that a cause-and-effect relationship cannot be confirmed.
\end{abstract}

Key words: oculogyric crisis, clebopride

(Inter Med 47: 551-552, 2008)

(DOI: 10.2169/internalmedicine.47.0623)

\section{Introduction}

Clebopride (4-amino-N-(1-benzyl-4-piperidyl)-5-chloro-Oanisamide) is a substituted benzamide which shares the antidopaminergic activities of the known benzamide derivative, metoclopramide (1). Clebopride has been shown to be effective and is currently available for the treatment of functional dyspepsia and vomiting (2-4). It is a relatively safe drug, with rare CNS side effects, such as the occurrence of parkinsonism and acute dystonia (5). However, the occurrence of an oculogyric crisis related to clebopride treatment has not been reported previously. Here, we present a young woman with an oculogyric crisis following the administration of clebopride.

\section{Case Report}

The patient was a 21-year-old woman who presented with repeated involuntary elevation of both eyes with retrocollis for 6 hours. Two weeks before admission, the patient had been treated for acute gastritis. Her medications included clebopride malate $0.68 \mathrm{mg}$ with simethicone $200 \mathrm{mg}$ (cumulative dose $28.56 \mathrm{mg}$ ), tiropramide $200 \mathrm{mg}$, and pantoprazol sodium sesquihydrate $45.1 \mathrm{mg}$, three times a day. There was no history of substance abuse or previous psychiatric treatment.

On admission to the hospital, the patient was alert and oriented, with a blood pressure of $120 / 70 \mathrm{mmHg}$, a regular heart beat of 74 beats $/ \mathrm{min}$, and a temperature of $36.8^{\circ} \mathrm{C}$.
Physical examination did not demonstrate any abnormal findings. The patient had spasmodic upward deviations of the eyes and retrocollis. The other cranial nerves were normal and there was no evidence of an extrapyramidal, pyramidal or sensory disturbance. The deep tendon reflexes in all extremities were symmetric and normoactive and both plantar reflexes were flexor.

The magnetic resonance imaging of the brain and electroencephalography did not reveal any abnormality. The symptoms were relieved completely shortly after intravenous injection of $2 \mathrm{mg}$ of lorazepam. All medications including clebopride were discontinued and the symptoms associated with the oculogyric crisis have not recurred during the three months of follow-up.

\section{Discussion}

Clebopride, a substituted benzamide with affinity for dopamine D2 receptors, acts on the hypothalamus (antagonism of apomorphine-induced hypothermia in mice), the chemoreceptor trigger zone of the area postrema (antagonism of apomorphine-induced vomiting in $\operatorname{dogs}$ ) and the mesolimbic system (6). It also blocks the dopaminergic receptors in the gastrointestinal tract (7). Originally, clebopride was selected for further development on the basis of its potency, which is ten times higher than that of metoclopramide, and possibly for its lack of drug-induced extrapyramidal symptoms $(1,8)$. However, chronic treatment with clebopride may be associated not only with reversible parkinsonian-like symptoms (involuntary movements of the 
tongue and mouth, blepharospasm, trismus, torticollis, resting tremor, rigidity and bradykinesia in both arms and legs), but also with tardive dyskinesia, which is potentially irreversible (5).

Oculogyric crisis is characterized by spasmodic deviations of the eyes. The direction of deviation is most commonly upward, though it may be lateral or uncommonly, downward or oblique. The duration of spasm may be from a few minutes to several hours. Convergence spasms have been described $(9,10)$. Oculogyric crisis is seen most commonly in association with phenothiazine toxicity and can occur after postencephalitic Parkinsonism. Seventy-two drugs have been reported as possible causes of oculogyric crisis (11). Although the mechanism of acute oculogyric crisis is uncertain, it is believed to be similar to that proposed for other dystonic reactions. The most plausible mechanisms suggest a compensatory dopamine release from presynaptic terminals in response to blockade of postsynaptic dopamine receptors, and upregulation or increased sensitivity of postsynaptic receptors in response to diminished quantities of dopamine or both $(12,13)$.

In summary, based on the temporal relationship of the administration of clebopride to the occurrence of the event and the absence of other possible etiologies, clebopride is implicated as the cause of this acute oculogyric crisis, despite the fact that a cause-and-effect relationship cannot be confirmed.

\section{Acknowledgement}

This study was supported by a grant of the Korea Health $21 \mathrm{R}$ $\& D$ Project, Ministry of Health and Welfare, Republic of Korea (project no. A060093).

\section{References}

1. Bleiberg H, Piccart M, Lips S, Panzer JM, N'Koua Mbon JB. A phase I trial of a new antiemetic drug-clebopride malate-in cisplatin-treated patients. Ann Oncol 3: 141-143, 1992.

2. Niemengeers CJ. Antiemetic specificity of dopamine antagonists. Psychopharmacology 78: 210-213, 1982.

3. Duarte DF, Linhares S, Gesser N, Pederneiras SG. Effect of intramuscular clebopride on postoperative nausea and vomiting. Clin Ther 7: 365-371, 1985.

4. Del Favero G, Di Mario F, Meggiato T, Scalon P, Mastropaolo G, Marin A. Efficacy and safety of clebopride in functional dydpepsia. Ital J Gastroenterol 23: 393, 1991.

5. Tonini M, Cipollina L, Poluzzi E, Crema F, Corazza GR, De Ponti F. Review article: clinical implications of enteric and central D2 receptor blockade by antidopaminergic gastrointestinal prokinetics. Aliment Pharmacol Ther 19: 379-390, 2004.

6. Roberts DJ. The pharmacological basis of the therapeutic activity of clebopride and related substituted benzamides. Curr Ther Res 31: S1-S44, 1982.

7. Roberts DJ. Modo Des accion del clebopride como bloqueante se- lectivo de los receptores dopaminicos perifericosy centrals. Rev Eps Enferm Apar Dig 1: 9-42, 1979 (in Spanish).

8. Bavestrello L, Caimi L, Barbera A. A double-blind comparison of clebopride and placebo in dyspepsia secondary to delayed gastric emptying. Clin Ther 7: 468-473, 1985.

9. Leigh RJ, Loley JM, Remler BF, Civil RH. Oculogyric crisis: a syndrome of thought disorder and ocular deviation. Ann Neurol 22: 12-17, 1987.

10. Jeong DS, Cho SG, Ahn MY, Sung KB. Oculogyric crisis after albendazole medication. J Korean Neurol Assoc 16: 421-422, 1998.

11. Fraunfelder FT, Fraunfelder FW, eds. Drug-induced Ocular Side Effects. 5th ed. Butterworth-Heinemann, Woburn, MA, 2001: 413415, 727-728.

12. Crismon ML, Dorson PG. Schizophrenia. In: Pharmacotherapy: A Pathophysiologic Approach. 2nd ed. DiPiro JT, Talbert RL, Hayes PE, Yee GC, Matzke GR, Posey LM, Eds. Elsevier, New York, 1992: 1030-1031.

13. Burstein AH, Fullerton T. Oculogyric crisis possibly related to pentazocine. Ann Pharmacother 27: 874-876, 1993.

(C) 2008 The Japanese Society of Internal Medicine http://www.naika.or.jp/imindex.html 